\title{
Radiomics-based machine learning analysis and characterization of breast lesions with multiparametric diffusion-weighted MR
}

\author{
Kun Sun ${ }^{1 \dagger}$, Zhicheng Jiao ${ }^{2 \dagger}{ }^{\text {, Hong Zhu }}{ }^{1}$, Weimin Chai ${ }^{1}, \mathrm{Xu} \mathrm{Yan}^{3}$, Caixia Fu ${ }^{4}$, Jie-Zhi Cheng ${ }^{5}$, Fuhua Yan ${ }^{*}$ (10 and \\ Dinggang Shen ${ }^{5,6^{*}}$
}

\begin{abstract}
Background: This study aimed to evaluate the utility of radiomics-based machine learning analysis with multiparametric DWI and to compare the diagnostic performance of radiomics features and mean diffusion metrics in the characterization of breast lesions.

Methods: This retrospective study included 542 lesions from February 2018 to November 2018. One hundred radiomics features were computed from mono-exponential (ME), biexponential (BE), stretched exponential (SE), and diffusion-kurtosis imaging (DKI). Radiomics-based analysis was performed by comparing four classifiers, including random forest (RF), principal component analysis (PCA), L1 regularization (L1R), and support vector machine (SVM). These four classifiers were trained on a training set with 271 patients via ten-fold cross-validation and tested on an independent testing set with 271 patients. The diagnostic performance of the mean diffusion metrics of $M E\left(\mathrm{mADC}_{\mathrm{all}}\right.$ $\left.b^{\prime}, \mathrm{mADC}_{0-1000}\right), \mathrm{BE}\left(\mathrm{mD}, \mathrm{mD}{ }^{*}, \mathrm{mf}\right), \mathrm{SE}(\mathrm{mDDC}, \mathrm{ma})$, and $\mathrm{DKI}(\mathrm{mK}, \mathrm{mD})$ were also calculated for comparison. The area under the receiver operating characteristic curve (AUC) was used to compare the diagnostic performance.
\end{abstract}

Results: RF attained higher AUCS than L1R, PCA and SVM. The AUCs of radiomics features for the differential diagnosis of breast lesions ranged from 0.80 (BE_D*) to 0.85 (BE_D). The AUCs of the mean diffusion metrics ranged from 0.54 (BE_mf) to 0.79 (ME_mADC $0-1000$ ). There were significant differences in the AUCs between the mean values of all diffusion metrics and radiomics features of AUCs (all $P<0.001$ ) for the differentiation of benign and malignant breast lesions. Of the radiomics features computed, the most important sequence was BE_D (AUC: 0.85), and the most important feature was FO-10 percentile (Feature Importance: 0.04).

Conclusions: The radiomics-based analysis of multiparametric DWI by RF enables better differentiation of benign and malignant breast lesions than the mean diffusion metrics.

Keywords: Breast cancer, Diffusion-weighted MRI, Machine learning, Random forest

*Correspondence: yfh11655@rjh.com.cn; Dinggang.Shen@gmail.com

${ }^{\dagger} K u n$ Sun and Zhicheng Jiao contributed equally to this work

1 Department of Radiology, Ruijin Hospital, Shanghai Jiaotong University School of Medicine, Shanghai, China

${ }^{5}$ Department of Research and Development, Shanghai United Imaging Intelligence Co., Ltd., Shanghai, China

Full list of author information is available at the end of the article

\section{Background}

Breast MRI is widely used for breast cancer diagnosis and treatment evaluation [1]. Dynamic contrast-enhanced (DCE) sequences with the use of a contrast agent can provide both morphological and hemodynamic cues for lesion diagnosis. However, a higher false-positive rate and background parenchymal enhancement limit the diagnostic specificity of DCE $[2,3]$.

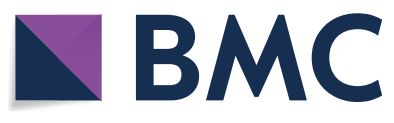

(c) The Author(s) 2021. Open Access This article is licensed under a Creative Commons Attribution 4.0 International License, which permits use, sharing, adaptation, distribution and reproduction in any medium or format, as long as you give appropriate credit to the original author(s) and the source, provide a link to the Creative Commons licence, and indicate if changes were made. The images or other third party material in this article are included in the article's Creative Commons licence, unless indicated otherwise in a credit line to the material. If material is not included in the article's Creative Commons licence and your intended use is not permitted by statutory regulation or exceeds the permitted use, you will need to obtain permission directly from the copyright holder. To view a copy of this licence, visit http://creativecommons.org/licenses/by/4.0/. The Creative Commons Public Domain Dedication waiver (http://creativeco mmons.org/publicdomain/zero/1.0/) applies to the data made available in this article, unless otherwise stated in a credit line to the data. 
Diffusion-weighted imaging (DWI), a noninvasive contrast agent-free method, has been established for breast MR imaging and could improve the diagnostic specificity of lesions suspicious for breast cancer $[4,5]$. Conventional DWI (mono-exponential model) with 2 to 3 b-values for the measurement of the apparent diffusion coefficient (ADC) is the most commonly used diffusion fitting model for the characterization of breast lesions [6]. Furthermore, several studies have suggested that biexponential (BE), stretched-exponential (SE), or diffusion kurtosis imaging (DKI), fitting with multi-b-value sequences, could provide more accurate information about water diffusion [7-10]. Le Bihan et al. [11, 12] proposed the intravoxel incoherent motion (IVIM) model, a kind of BE fitting, to separately calculate fast and slow diffusion components. The SE model was introduced by Bennett et al. [13] to depict the heterogeneity of intravoxel diffusion rates and the distributed diffusion effect. The DKI model was proposed by Jensen et al. [14] to reflect the complexity of the microenvironment. Since DWI with different fitting models may demonstrate different aspects of tissue properties [7, 15, 16], informative radiomics features could be derived from these models to better characterize breast lesions.

Radiomics-based analysis profiles lesions with extensive morphological and textural features for latter classification models to attain better differential diagnosis, prognosis prediction, and tumor subtype diagnosis, etc. [17-20]. Previous radiomics studies [21-23] of breast lesions focused more on the modalities of T2WI and DCE. Fewer studies [17, 24, 25] have considered the value of multiparametric DWI. However, to our knowledge, no study comparing the radiomics features of these different diffusion imaging approaches in the differentiation of breast lesions has been conducted. Further study of multiparametric DWI using more effective machine learning methods is needed to better understand their predictive value in breast cancer diagnosis.

We hypothesized that the diagnostic accuracy of breast lesions using multi-b-value sequences combined with $\mathrm{ME}, \mathrm{BE}, \mathrm{SE}$ and DKI can be improved by radiomicsbased analysis. The purpose of this study is to compare radiomics features and the mean values of diffusion metrics in the assessment of breast lesions with four machine learning methods, i.e., random forest (RF), L1 regularization combined with linear regression (L1R-LR), principal component analysis combined with linear regression (PCA-LR), and support vector machine (SVM).

\section{Methods}

\section{Study design and patient selection}

This retrospective study was performed with a prospectively acquired data set with institutional and governmental review board approval. The local Institutional Review Board (IRB) approved this study. Written informed consent was obtained from each participant. From February 2018 to November 2018, 622 women with lesions suspicious for breast cancer on mammography or ultrasonography (i.e., BI-RADS category 4 or 5) underwent MRI examinations with multi-b DWI. The exclusion criteria included the following: patients previously treated for a malignancy $(N=13)$, patients without histopathological results $(N=27)$, and patients with motion artifacts $(N=5)$, lesions that were not seen in DWI mappings $(N=25)$, and the mean value of the goodness-of-fit of the diffusion fitting model was less than $0.8(N=10)$. Ultimately, a total of 542 women (mean age, 51 years; age range, 24-84 years), with 542 lesions were enrolled in this study.

\section{MR imaging}

All breast MRI examinations were performed on a $1.5 \mathrm{~T}$ MR scanner (MAGNETOM Aera, Siemens Healthcare, Erlangen, Germany) with a dedicated 18-channel phasedarray breast coil. The breast MR examinations included fat-suppressed T2-weighted fast spin-echo imaging, T1-weighted imaging (T1WI), DWI, and DCE T1WI. All MR imaging examinations were performed before biopsy. The parameters of the above sequences are shown in Additional file 1: Appendix S1.

\section{Image postprocessing and lesion segmentation}

After data acquisition, all images were transferred to N4ITK for the data normalization. Then, all these data were assessed by KS and WC (with 8 years and 12 years of experience in breast imaging) to identify all lesions by using the DWI source images with b values of $1000 \mathrm{~s} /$ $\mathrm{mm}^{2}$, T2-weighted images, and the first phase of postcontrast T1-weighted images. Clinical information and the X-ray and US images were provided to the radiologists. The lesions were manually segmented in the DW images $\left(\mathrm{b}_{1000}\right)$ on all visible sections, resulting in a threedimensional image of the lesion. Lesions were segmented by using the inner border of the lesion to minimize partial volume effects. All volumes of interest (VOIs) were manually segmented and labeled via a free open-source software package (ITK-SNAP, version 3.4.0, http://www. itksnap.org). An overview of our workflow is illustrated in Fig. 1.

\section{Diffusion data analysis and processing}

All diffusion parameter maps were generated using an in-house MATLAB software (MathWorks, Natick, MA, USA). The software first applied a Gaussian filter with a full width at a half maximum of $3 \mathrm{~mm}$ to suppress noise 


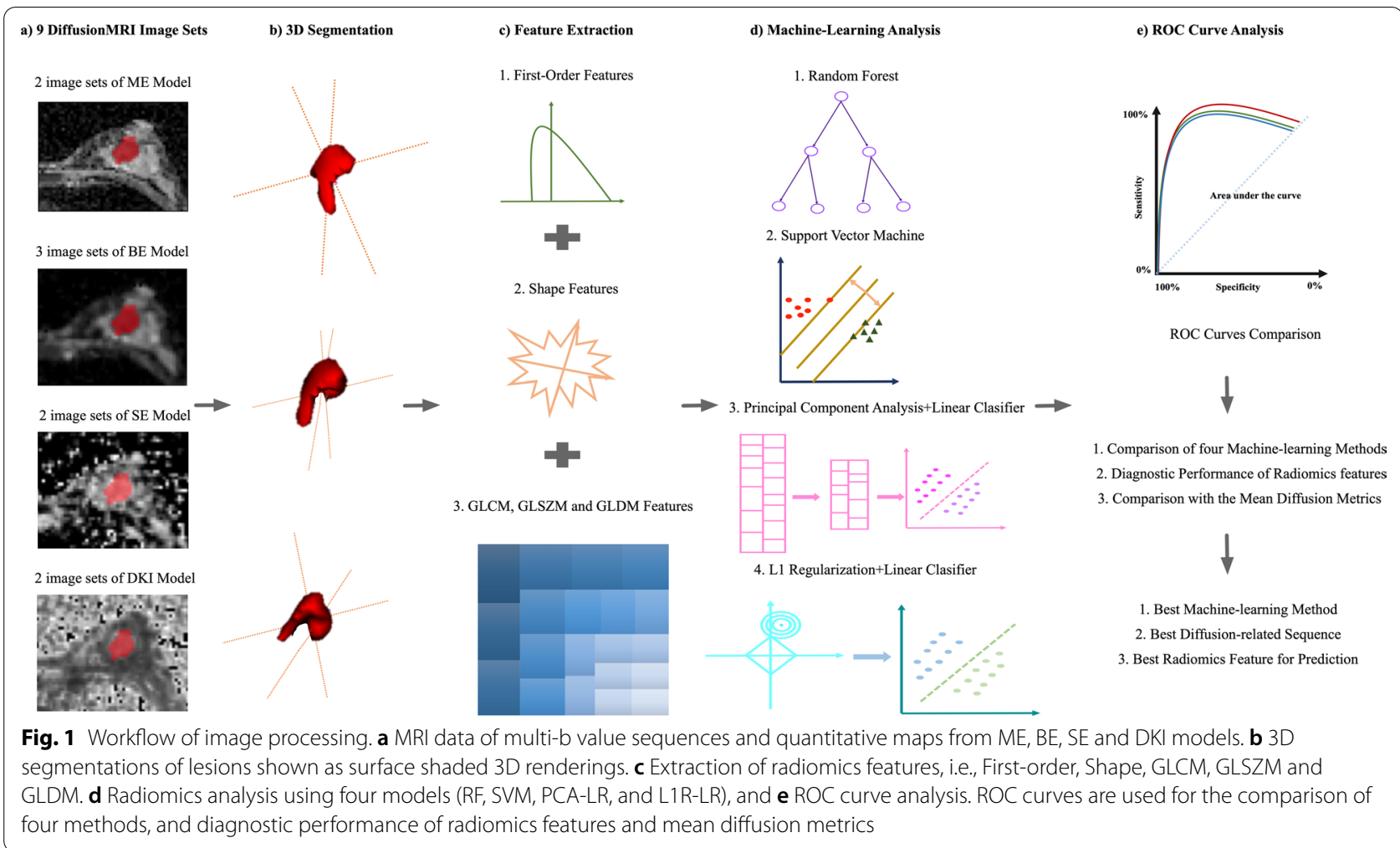

in the diffusion images before the pixel-by-pixel fitting process. Four diffusion models are described as follows:

\section{ME model}

ADC maps were generated according to the following equation:

$$
S_{b} / S_{0}=\exp (-b \cdot A D C),
$$

where $S_{b}$ represents signal intensity in the presence of diffusion sensitization, and $S_{0}$ represents signal intensity in the absence of diffusion sensitization. The $\mathrm{ADC}_{\text {all-b }}$ maps were generated by using all $13 \mathrm{~b}$ values. The $\mathrm{ADC}_{0-1000}$ maps were generated by using $\mathrm{b}$ values of 0 and 1000 .

2. BE_IVIM model

The IVIM parameters were fitted using the following IVIM model (proposed by Le Bihan et al. [11, 12]:

$$
S_{b} / S_{0}=(1-f) \cdot \exp (-b \cdot D)+f \cdot \exp \left(-b \cdot D^{*}\right),
$$

where $D$ is the true diffusion as reflected by the pure molecular diffusion, $f$ is the fractional perfusion related to microcirculation, and $D^{*}$ is the pseudodiffusion coefficient that represents perfusion-related diffusion or incoherent microcirculation.

\section{SE model}

The SE model was used to obtain the molecular water diffusion heterogeneity index $(\alpha)$ and the distributed diffusion coefficient $(D D C)$ through the following equation:

$$
S_{b} / S_{0}=\exp \left[-(b \cdot D D C)^{\alpha}\right],
$$

where $\alpha$ is related to the intravoxel molecular water diffusion heterogeneity, which ranges from 0 to 1 . A numerically high $\alpha$ value represents low intravoxel diffusion heterogeneity (approaching mono-exponential decay). $D D C$ represents the mean intravoxel diffusion rate.

4. DKI model

Calculation of DKI parameters was performed by fitting the following nonlinear equation:

$$
S_{b} / S_{0}=\exp \left(-b \cdot D+1 / 6 \cdot b^{2} \cdot D^{2} \cdot K\right),
$$

where $K$ is a unitless parameter that quantifies the deviation of water motion from the Gaussian distribution. $K$ is zero for a perfect Gaussian diffusion, and a large $K$ indicates considerable deviation of diffusion from a perfect Gaussian behaviour. $D$ is a corrected $A D C$ by removing non-Gaussian bias. 


\section{Feature extraction}

Radiomics features were calculated using the PyRadiomics Python package (version 2.1.2), and the recommended default settings were used for the analysis [26]. Each map extracted 100 features comprising 18 firstorder (FO) features, 14 shape features, 22 Gy level cooccurrence matrix (GLCM) features, 16 Gy level run length matrix (GLRLM) features, 16 Gy level size zone matrix (GLSZM) features, and 14 Gy level dependence matrix (GLDM) features. Details of the extracted features are shown in Additional file 1: Appendix S2. In total, 900 features were extracted. The interclass correlation coefficients (ICCs) were used to determine the interobserver reproducibility of the radiomics features [27].

The mean diffusion metrics of $\mathrm{ME} \mathrm{(mADC} \mathrm{m}_{\mathrm{all}-\mathrm{b}}$, $\left.\mathrm{mADC}_{0-1000}\right), \mathrm{BE}\left(\mathrm{mD}, \mathrm{mD}^{*}, \mathrm{mf}\right), \mathrm{SE}(\mathrm{mDDC}, \mathrm{m \alpha})$, and DKI $(\mathrm{mK}, \mathrm{mD})$ were extracted from the radiomics set for separate analysis. Feature importance (FI) was calculated by using random forest. Feature importance was determined as the mean decrease in the impurity of the random forest as previously described [28].

\section{RF, L1R, PCA, and SVM}

The 542 subjects were randomly and equally divided into a training set containing 271 subjects and an independent testing set containing the remaining 271 subjects. The ratios of malignant and benign subjects in the training set and the testing set were equal to the ratio in the whole dataset. The RF, SVM, PCA-LR, and L1R-LR algorithms were all based on the most widely used machine learning Python package, i.e., Scikit-learn [29]. For RF, the parameters were set as the default values, the number of trees was 100, and the maximum depth of the tree was 3. For L1 regularization (L1R), the features were selected implicitly by the $\mathrm{L} 1$ regularization of the linear classifier. L1R enforced the coefficients of the linear model to be sparse, thus making a small subset of radiomics features contribute to the final results. For PCA, 100 features were selected based on their power to differentiate benign from malignant lesions in the training set by sorting the lowest $P$ values. Then, the first 10 principal components were chosen for the linear model for prediction. The parameter settings of both PCA and L1R followed the widely-used strategies in other MRI-based radiomics studies for breast cancer [23]. For SVM, we used the radial basis function (RBF) kernel. The parameters were optimized with respect to the training set. The hyperparameters of the above four methods are shown in Additional file 1: Appendix S3. The classifiers were trained using the repeated tenfold cross-validation (CV) method (100 times) in the training cohort, and their prognostic performance was then evaluated in the validation cohort using the area under the receiver operating characteristic
(ROC) curve. A more detailed description of the frequencies of the features of RF during 100 times of tenfold CV is shown in Additional file 1: Appendix S4.

\section{Statistical analysis}

A goodness-of fit evaluation was performed for fitting of the BE, SE and DKI models by using MATLAB (MathWorks). The $\mathrm{R}^{2}$ value was calculated [9]. ROC curves were generated for the mean diffusion metrics (ME$\mathrm{mADC}_{\text {all b}}, \mathrm{ME}-\mathrm{mADC} \mathrm{C}_{0-1000}, \mathrm{BE}-\mathrm{mD}, \mathrm{BE}-\mathrm{mD}$, BE-mf, SE_mDDC, SE_m $\alpha$, DKI-mK, and DKI-mD), and the ROC curves of all the 9 DWI image sets of the RF, L1R, PCA, and SVM models were calculated for comparison. The ROC curves of the 9 diffusion-related image sets were calculated from the results obtained by the $\mathrm{CV}$ models in the independent testing set. To compare the AUCs of the mean diffusion metrics and radiomics features, the McNemar test was used for the paired cases. All these comparisons were run 100 times, and we obtained the mean $P$ values. Bonferroni adjustment was performed to control for $\alpha$ error inflation [29]. A $P$ value less than $0.05 / 23(0.00217)$ was regarded as a significant difference. All statistical evaluations were performed by using software developed either with the Python programming language [30] or with MATLAB software.

\section{Results}

\section{Image quality of multi-b diffusion weighted imaging}

The mean $\mathrm{R}^{2}$ value for the BE model fit was $0.90 \pm 0.06$. The mean $\mathrm{R}^{2}$ value for the SE model fit was $0.95 \pm 0.03$. The mean $\mathrm{R}^{2}$ value for the DKI model fit was $0.99 \pm 0.01$.

The signal intensity of malignant lesions on the map of $b_{2500}$ was 113 . $25 \pm 31.53$. The signal intensity of benign lesions on the map of $b_{2500}$ was $36.83 \pm 10.73$. The signal to noise ratio (SNR) of $b_{2500}$ was $30.01 \pm 10.16$. The contrast noise ratio (CNR) of $b_{2500}$ was $2.25 \pm 0.67$. The lesion contrast on the map of $b_{2500}$ was $3.20 \pm 1.04$. A case of 23 datasets is shown in Additional file 1: Appendix S5.

\section{Patient demographic characteristics}

There was significant difference in demographic characteristics between patients with malignant lesions and patients with benign lesions $(55.0 \pm 12.2$ vs. $50.3 \pm 11.6$, $P<0.001)$.

\section{Pathological features}

Of the 542 lesions, 333 were malignant, and 209 were benign. The malignant lesions included ductal carcinoma in situ $(N=28)$, lobular carcinoma in situ $(N=1)$, invasive carcinoma $(N=274)$, invasive lobular carcinoma $(N=1)$, invasive solid papillary carcinoma $(N=9)$, malignant phyllodes tumors $(N=3)$, mucinous carcinoma $(N=8)$, metaplastic cancer $(N=1)$, diffuse large B-cell 
lymphoma $(N=2)$, encapsulated papillary carcinoma $(N=3)$, and invasive micropapillary carcinoma $(N=3)$. Benign lesions included fibroadenoma $(\mathrm{N}=101)$, benign phyllodes tumors $(N=3)$, fibrocystic change $(N=4)$, cyst combined chronic infection $(N=6)$, papilloma $(N=54)$, usual ductal hyperplasia $(N=16)$, fat necrosis $(N=1)$, and adenosis $(N=24)$.

\section{Comparison of RF, L1R-LR, PCA-LR, and SVM in the diagnosis of breast lesions with multi-b diffusion-weighted imaging}

The AUCs of RF in the differential diagnosis of breast lesions ranged from 0.80 (BE_D*) to 0.85 (BE_D), whereas the AUCs of PCA-LR ranged from 0.53 (SE_DDC) to 0.78

Table 1 Comparisons between radiomics and mean diffusion metrics

\begin{tabular}{|c|c|c|c|}
\hline Maps & AUC of mean metrics & AUC of Radiomics & $P$ value \\
\hline \multicolumn{4}{|l|}{ ME } \\
\hline$m A D C_{0-1000}$ & $0.79(0.76-0.83)$ & $0.83(0.80-0.87)$ & 0.002 \\
\hline$m A D C_{a l l-b}$ & $0.77(0.73-0.81)$ & $0.84(0.80-0.88)$ & 0.001 \\
\hline \multicolumn{4}{|l|}{ BE_IVIM } \\
\hline $\mathrm{mD}$ & $0.75(0.72-0.79)$ & $0.85(0.81-0.89)$ & $<0.001$ \\
\hline$m D^{*}$ & $0.67(0.63-0.71)$ & $0.80(0.74-0.83)$ & $<0.001$ \\
\hline $\mathrm{mf}$ & $0.54(0.50-0.58)$ & $0.82(0.77-0.86)$ & $<0.001$ \\
\hline \multicolumn{4}{|l|}{ SE } \\
\hline $\mathrm{mDDC}$ & $0.77(0.74-0.81)$ & $0.81(0.77-0.85)$ & 0.030 \\
\hline $\mathrm{ma}$ & $0.62(0.58-0.66)$ & $0.80(0.77-0.84)$ & $<0.001$ \\
\hline \multicolumn{4}{|l|}{ DKI } \\
\hline $\mathrm{mD}$ & $0.78(0.73-0.80)$ & $0.83(0.80-0.88)$ & 0.005 \\
\hline $\mathrm{mK}$ & $0.75(0.71-0.80)$ & $0.84(0.80-0.88)$ & 0.001 \\
\hline \multicolumn{4}{|c|}{ 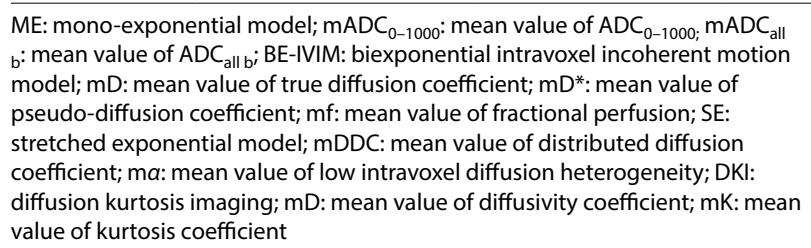 } \\
\hline
\end{tabular}

(BE_D*). The AUCs of L1R-LR and SVM ranged from 0.53 (SE_DDC) to $0.83\left(\mathrm{ME}_{-} \mathrm{ADC}_{0-1000}\right)$ and from 0.51 (SE_DDC) to 0.81 (ME_ADC ${ }_{0-1000}$ ), respectively.

The top image image sets with the highest AUCs by the RF were BE_D (0.85), ME_ADC all b $_{\text {b }}(0.84)$, DKI_K $(0.84)$, ME_ADC $_{0-1000}(0.83)$ and DKI_D (0.83). The results of all AUCs by RF are shown in Table 1. The top five image sets with the highest mean AUCs were ME_ADC ${ }_{0-1000}$ (0.81), BE_D (0.81), ME_ADC all b $_{\text {b }}(0.81)$, DKI_D $(0.80)$, and DKI_K $(0.80)$.

Details on the top five image sets with the highest mean AUCs by RF, SVM, L1R-LR, and PCA-LR are shown in Table 2. The comparisons between RF and L1R, and between PCA and SVM are shown in Additional file 1: Appendix S6.

RF achieved the highest frequency of the highest AUCs compared with L1R-LR, PCA-LR, and SVM (8/9 vs. 1/9 vs. $0 / 9$ vs. $0 / 9, P<0.001)$. The mean AUCs of the nine image sets by RF, L1R-LR, PCA-LR and SVM were 0.82 , $0.78,0.73$, and 0.76 , respectively.

\section{Diagnostic performance comparison of radiomics features by RF and the mean values of diffusion metrics}

The interobserver reproducibility of radiomics feature extraction was satisfactory, with ICCs greater than 0.80 for all extracted features. The AUCs of the radiomics features for the differential diagnosis of breast lesions ranged from 0.80 (BE_D*) to 0.85 (BE_D), with a sensitivity of $83 \%$ to $88 \%$, and a specificity of $74 \%$ to $82 \%$. The AUCs of the mean diffusion metrics ranged from 0.54 (BE_mf) to 0.79 (ME_mADC ${ }_{0-1000}$ ), with a sensitivity of $74 \%$ to $88 \%$, and a specificity of $41 \%$ to $71 \%$. The AUCs of the radiomics features for the differential diagnosis of breast lesions were higher than those of the corresponding mean diffusion metrics, and there were significant differences in the AUCs between the mean values of the diffusion metrics $\left(\mathrm{ME} \_\mathrm{mADC}\right.$ all-b, $\mathrm{ME}_{-} \mathrm{mADC}_{0-1000}$, BE_mD, BE_mD", BE_mf, SE_m $\alpha$, and DKI_mK) and the corresponding radiomics features of AUCs $($ all $P<0.002)$

Table 2 Diagnostic performance of ME_ADC ${ }_{0-1000}$, BE_IVIM_D, ME_ADC all b, DKI-D and DKI-K by using RF, L1R-LR, PCA-LR, and SVM, respectively

\begin{tabular}{|c|c|c|c|c|c|}
\hline \multirow[t]{2}{*}{ Maps } & \multicolumn{5}{|l|}{ AUC $(95 \% \mathrm{CI})$} \\
\hline & RF & L1R-LR & PCA-LR & SVM & mAUC \\
\hline ME-ADC $0-1000$ & $0.83(0.80-0.87)$ & $0.83(0.79-0.87)$ & $0.76(0.70-0.81)$ & $0.81(0.76-0.85)$ & 0.81 \\
\hline BE-IVIM-D & $0.85(0.81-0.89)$ & $0.83(0.78-0.87)$ & $0.75(0.70-0.82)$ & $0.80(0.75-0.85)$ & 0.81 \\
\hline$M E-A D C_{\text {all b }}$ & $0.84(0.80-0.87)$ & $0.82(0.79-0.87)$ & $0.77(0.70-0.83)$ & $0.79(0.74-0.85)$ & 0.81 \\
\hline DKI-D & $0.83(0.80-0.86)$ & $0.83(0.78-0.86)$ & $0.75(0.74-0.82)$ & $0.80(0.77-0.84)$ & 0.80 \\
\hline DKI-K & $0.84(0.81-0.89)$ & $0.83(0.78-0.87)$ & $0.74(0.70-0.80)$ & $0.79(0.75-0.85)$ & 0.80 \\
\hline
\end{tabular}

RF: random forest; SVM: support vector machine; PCA: principal component analysis; L1R: L1 regularization; LR: linear regression; mAUC: mean values of AUCs of RF, L1R-LR, PCA-LR and SVM 
for the differentiation of benign and malignant breast lesions. Details of the comparison are shown in Table 3.

\section{Importance of diffusion-related radiomics features}

Details of all radiomics feature importance were shown in Additional file 1: Appendix S7. Regarding the radiomics features computed from nine image sets, the top five important features were $\mathrm{FO}-10$ percentile $(\mathrm{FI}=0.043)$, FOMedian $(\mathrm{FI}=0.030)$, Shape-Sphericity $(\mathrm{FI}=0.030)$, FOSkewness $(\mathrm{FI}=0.029)$, and Shape-Flatness $(\mathrm{FI}=0.026)$.

Of the radiomics features computed from the map of BE_IVIM_D, which had the highest AUC (0.85), the top five most important features were FO-10 percentile $(\mathrm{FI}=0.07)$, FO-Skewness $(\mathrm{FI}=0.06)$, FO-Minimum $(\mathrm{FI}=0.04)$, GLCM-Cluster Shade $(\mathrm{FI}=0.04)$, and FOMedian $(F I=0.02)$. Details of the top 20 important features of BE_IVIM_D are shown in Fig. 2. The ROC curve of BE_IVIM_D is shown in Fig. 3.

\section{Discussion}

Based on experimental results of this study, the $\mathrm{BE}_{-}$ IVIM_D map (with the highest AUC by RF), and the FO-10 percentile feature (with the highest FI by RF) from the radiomics-based analysis of multiparametric DWI are recommended in the characterization of breast lesions. Furthermore, we also found that the diagnostic performance of multiparametric DWI-derived radiomics was superior to that of the mean diffusion metrics in differentiating between benign and malignant breast lesions. This finding suggests that the radiomics-based analysis for multiparametric DWI has a potentiallyimproved performance in the classifications of breast lesions.
The majority of radiomics-based analyses in breast MRI research utilize T2WI, contrast T1WI, and conventional DWI [17, 18, 31, 32]. To the best of our knowledge, this is the first study that extensively explored radiomics from multi-b-value maps and its commonly used fitting models (ME, BE, SE, and DKI), which could reflect more details of both Gaussian and non-Gaussian water diffusion distributions in tumors. Bickelhaupt et al. [17] demonstrated that the radiomics features of DKI can help differentiate malignant breast lesions from benign lesions. However, they only used the fitting model of DKI, and their scan sequences contained both the single-shot echo planar imaging (ss-EPI) in 95 patients and readout-segmented echo-planar imaging (rs-EPI) in 127 patients. We used four clinically used diffusion fitting models, and we also enlarged the sample size (542 lesions) in our study. Moreover, all the patients in our study were scanned with rs-EPI, which has significantly higher image quality and lesion conspicuity than ss-EPI, as suggested by previous studies [33, 34].

Many radiomics-based machine learning methods can be used for lesion classification [17, 18, 35, 36]. In this study, we extensively explored four promising algorithms of RF, L1R-LR, PCA-LR and SVM, which have been demonstrated to have high effectiveness in the previous radiomics studies [23, 37]. We found that the $\mathrm{ADC}_{0-1000}$ feature attained the highest mean AUC with all four algorithms, indicating that the mono-exponential model had already provided enough diagnostic information for breast cancer. Furthermore, RF had the highest probability of achieving the highest AUCs (8/9). Accordingly, this finding further corroborates the robustness and strong generalization power of RF [28]. Thus, in our further

Table 3 Diagnostic performance of multi-b diffusion maps based on ME, BE, SE and DKI models

\begin{tabular}{|c|c|c|c|c|c|}
\hline Maps & AUC $(95 \% \mathrm{Cl})$ & Sensitivity\% $(95 \% \mathrm{Cl})$ & Specificity\% $(95 \% \mathrm{Cl})$ & PPV\% (95\% Cl) & NPV\% $(95 \% \mathrm{Cl})$ \\
\hline \multicolumn{6}{|l|}{ ME } \\
\hline $\mathrm{ADC}_{0-1000}$ & $0.83(0.80-0.87)$ & 88 (82-93) & $79(68-87)$ & $87(81-92)$ & $80(72-88)$ \\
\hline$A D C_{\text {all-b }}$ & $0.84(0.80-0.88)$ & 88 (82-93) & 81 (71-90) & $88(82-94)$ & $80(72-88)$ \\
\hline \multicolumn{6}{|l|}{ BE-IVIM } \\
\hline D & $0.85(0.81-0.89)$ & $88(81-93)$ & $82(73-90)$ & 89 (83-93) & $80(72-89)$ \\
\hline$D^{*}$ & $0.80(0.74-0.83)$ & $83(76-88)$ & $77(66-86)$ & $85(77-91)$ & $73(65-81)$ \\
\hline f & $0.82(0.77-0.86)$ & 85 (79-90) & $79(68-87)$ & $86(80-92)$ & $77(68-84)$ \\
\hline \multicolumn{6}{|l|}{ SE } \\
\hline DDC & $0.81(0.77-0.85)$ & $85(78-90)$ & $77(66-86)$ & 85 (79-91) & $76(68-84)$ \\
\hline$a$ & $0.80(0.77-0.84)$ & $85(79-89)$ & $76(67-86)$ & 85 (80-91) & 76 (69-82) \\
\hline \multicolumn{6}{|l|}{ DKI } \\
\hline $\mathrm{D}$ & $0.83(0.80-0.88)$ & 87 (80-94) & 79 (69-88) & $87(81-93)$ & $80(71-89)$ \\
\hline K & $0.84(0.80-0.88)$ & 87 (80-92) & $81(72-89)$ & 88 (83-93) & $79(72-86)$ \\
\hline
\end{tabular}



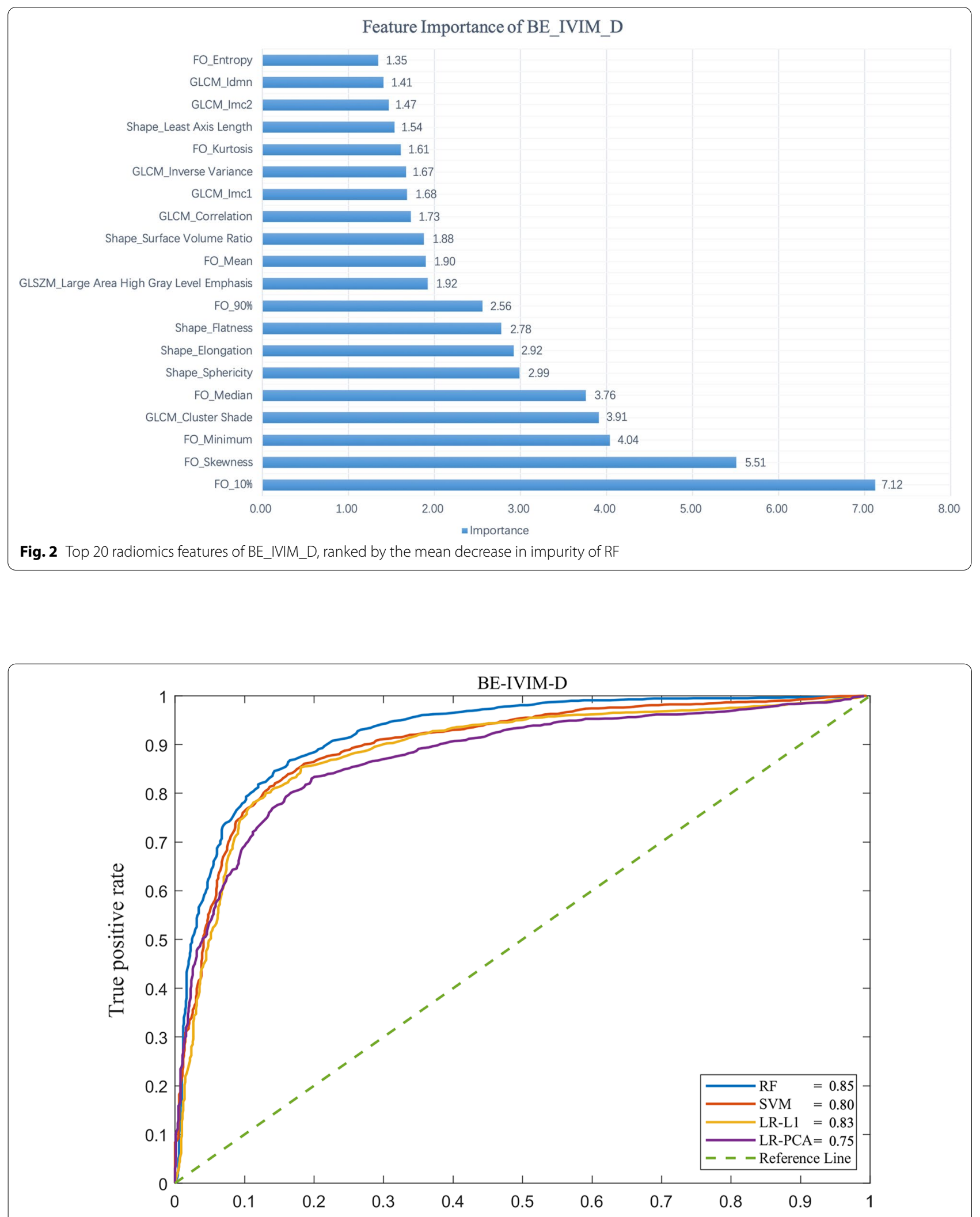

False positive rate

Fig. 3 ROC curve analysis of BE_IVIM_D for radiomics-based analysis with RF, L1R, PCA, and SVM, respectively 
analysis, both the calculation of feature importance and the comparison of AUCs were based on the results of RF.

The most predictive image set by RF (i.e., with the highest AUC, sensitivity and specificity) was BE_IVIM_D. Of note, BE_IVIM_D can remove the influence of perfusion and therefore reflects the true diffusion coefficient, better reflecting water movement in the living tissues. This may be the reason why the radiomics features computed from BE_IVIM_D provide more accurate information on water diffusion in breast cancer classification. Furthermore, the most important radiomics feature of BE_IVIM_D is the FO-10-percentile. Unlike that in previously reported studies [38-40], our experimental results did not suggest that texture features can attain better performance than the FO features on BE_IVIM_D. Accordingly, the FO features may be more predictive of lesion malignancy. On the other hand, the FO-10-percentile was also shown to be the most important feature $(\mathrm{FI}=0.043)$ for the differentiation of benign and malignant breast lesions, indicating that first-order features remain important cues in multiparametric DWI for the differential diagnosis of breast lesions.

Our study has several limitations. First, all lesions in this study were drawn manually, which was time-consuming. Thus, automated lesion segmentation will be implemented in our future study to improve the objectiveness of lesion boundaries and to expedite preprocessing. Second, our multi-b value sequences were acquired with a fixed protocol, whereas the choice of optimal b-values could vary across different institutions. Thus, there was a lack of an external independent verification dataset to verify the generalization ability of this study's findings. Finally, this study employed all extracted diffusion-related radiomics for breast cancer diagnosis. The feature selection strategy was not implemented in this study. In future studies, we will conduct feature selection to optimize the construction of radiomics models.

\section{Conclusions}

In conclusion, the BE_IVIM_D map, and of FO-10-percentile feature by RF enabled accurate differentiation between malignant and benign breast lesions. Radiomics features computed from multiparametric DWI performed better than the mean values in distinguishing benign and malignant breast lesions. Hence, our study may shed a light on the applicability of radiomics from the multiparametric DWI for the clinical diagnosis of breast lesions.

\section{Abbreviations}

DWI: Diffusion weighted imaging; ADC: Apparent diffusion coefficient; ROC: Receiver operating characteristic; AUC: Area under the ROC curve; BI-RADS: Breast imaging reporting and data system; Ss-EPI: Single-shot echo planar imaging; RS-EPI: Readout segmented echo planar imaging; ME: Mono exponential model; $M E \_A D C_{0-1000}: A C_{0-1000}$ map of $M E$ model; $\mathrm{MADC}_{0-1000}$ : Mean value of $\mathrm{ADC}_{0-1000}$; $\mathrm{BE}$ : Bi exponential model; $\mathrm{D}^{*}$ : Pseudo-diffusion coefficient; D: True diffusion coefficient; f: Fractional perfusion; BE_D*: D* map of BE model; $\mathrm{mD}^{*}$ : Mean value of $\mathrm{D}^{*}$; SE: Stretched exponential model; DDC: Distributed diffusion coefficient; $a$ : Low intravoxel diffusion heterogeneity; SE_DDC: DDC map of SE model; mDDC: Mean values of DDC; Cl: Confidence interval; DKI: Diffusion kurtosis imaging; K: Kurtosis coefficient; D: Diffusivity coefficient; DKI_K: Kurtosis map based on DKI model; DKI_D: Diffusivity map based on DKI model; mK: Mean value of kurtosis coefficient; mD: Mean value of diffusivity coefficient; IVIM: Intravoxel incoherent motion; RF: Random forest; SVM: Support vector machine; PCA: Principal component analysis; L1R: L1 regularization; LR: Linear regression; FO: First order; DCE: Dynamic contrastenhancement; T2WI:T2-weighted imaging; T1WI:T1-weighted imaging; IRB: Institutional review board; GLCM: Gray level co-occurrence matrix; GLRLM: Gray level run length matrix; GLSZM: Gray level size zone matrix; GLDM: Gray level dependence matrix; ICC: Interclass correlation coefficient; RBF: Radial basis function; CV: Cross validation; SNR: Signal to noise ratio; CNR: Contrast noise ratio; FI: Feature importance; PPV: Positive predictive value; NPV: Negative predictive value.

\section{Supplementary Information}

The online version contains supplementary material available at https://doi. org/10.1186/s12967-021-03117-5.

Additional file 1. The scanning parameters of T2WI, multi-b DWI, precontrast T1WI, and DCE T1WI.

\section{Acknowledgements}

Not applicable.

\section{Authors' contributions}

Literature search: KS, ZJ and HZ; Study design: KS, FY, and DS; Data collection: KS, HZ and WC; Data analysis: KS, and ZJ; Manuscript editing: WC, XY, CF and JC; Manuscript review: FY, and DS. All authors read and approved the final manuscript.

\section{Funding}

This study was funded by the National Natural Science Foundation of China (young scientists fund No.81801651).

\section{Availability of data and materials}

The datasets used or analyzed during the current study are available from the corresponding author on reasonable request.

\section{Declarations}

\section{Ethics approval and consent to participate}

All procedures performed in studies involving human participants were in accordance with the ethical standards of Ruijin Hospital. Informed consent was obtained from all individual participants included in the study.

\section{Consent for publication \\ Not applicable.}

\section{Competing interests}

We declare that KS and ZJ contributed equally to this work. We declare that $\mathrm{FY}$ and DS are all the corresponding authors. We declare that two co-authors (XY, and CF) are employees of Siemens Healthcare, and two co-authors (JC and DS) are employees of Shanghai United Imaging Intelligence. Only those authors who are not employees of above two companies (KS, ZJ, HZ, WC, FY) had control of the data and information submitted for publication.

\section{Author details}

${ }^{1}$ Department of Radiology, Ruijin Hospital, Shanghai Jiaotong University School of Medicine, Shanghai, China. ${ }^{2}$ Department of Diagnostic Imaging, Alpert Medical School of Brown University, Providence, USA. ${ }^{3}$ Scientific 
Marketing, Siemens Shanghai Magnetic Resonance Ltd., Shanghai, China. ${ }^{4}$ MR Application Development, Siemens Shenzhen Magnetic Resonance Ltd. Shenzhen, China. ${ }^{5}$ Department of Research and Development, Shanghai United Imaging Intelligence Co., Ltd., Shanghai, China. ${ }^{6}$ School of BME, Shanghai Tech University, Shanghai, China.

\section{Received: 24 July 2021 Accepted: 13 October 2021} Published online: 24 October 2021

\section{References}

1. Kuhl CK, Jost P, Morakkabati N, Zivanovic O, Schild HH, Gieseke J. Contrast-enhanced MR imaging of the breast at 3.0 and $1.5 \mathrm{~T}$ in the same patients: initial experience. Radiology. 2006;239:666-776.

2. Uematsu T, Kasami M, Watanabe J. Does the degree of background enhancement in breast MRI affect the detection and staging of breast cancer? Eur Radiol. 2011:21:2261-7.

3. Giess CS, Yeh ED, Raza S, Birdwell RL. Background parenchymal enhancement at breast MR imaging: normal patterns, diagnostic challenges, and potential for false-positive and false-negative interpretation. Radiographics. 2014;34:234-47.

4. Song SE, Park EK, Cho KR, Seo BK, Woo OH, Jung SP. Additional value of diffusion-weighted imaging to evaluate multifocal and multicentric breast cancer detected using pre-operative breast MRI. Eur Radiol. 2017;27:4819-27.

5. Spick C, Pinker-Domenig K, Rudas M, Helbich TH, Baltzer PA. MRI-only lesions: application of diffusion-weighted imaging obviates unnecessary MR-guided breast biopsies. Eur Radiol. 2014;24:1204-10.

6. Kul S, Cansu A, Alhan E, Dinc H, Gunes G, Reis A. Contribution of diffusion-weighted imaging to dynamic contrast-enhanced MRI in the characterization of breast tumors. Am J Roentgenol. 2011;196:210-7.

7. Liu C, Liang C, Liu Z, Zhang S, Huang B. Intravoxel incoherent motion (IVIM) in evaluation of breast lesions: comparison with conventional DWI. Eur J Radiol. 2013:82:e782-9.

8. Lai V, Lee VH, Lam KO, Sze HC, Chan Q, Khong PL. Intravoxel water diffusion heterogeneity MR imaging of nasopharyngeal carcinoma using stretched exponential diffusion model. Eur Radiol. 2015;25:1708-13.

9. Sun K, Chen X, Chai W, Fei X, Fu C, Yan X. Breast cancer: diffusion kurtosis MR imaging-diagnostic accuracy and correlation with clinicalpathologic factors. Radiology. 2015;277:46-55.

10. Suo S, Yin Y, Geng X, Zhang D, Hua J, Cheng F, et al. Diffusion-weighted MRI for predicting pathologic response to neoadjuvant chemotherapy in breast cancer: evaluation with mono-, bi-, and stretched-exponential models. J Transl Med. 2021;19:1-12.

11. Le Bihan D, Breton E, Lallemand D, Aubin ML, Vignaud J, Laval-Jeantet M. Separation of diffusion and perfusion in intravoxel incoherent motion MR imaging. Radiology. 1988;168:497-505.

12. Le Bihan D, Turner R, MacFall JR. Effects of intravoxel incoherent motions (IVIM) in steady-state free precession (SSFP) imaging: application to molecular diffusion imaging. Magn Reson Med. 1989;10:324-37.

13. Bennett KM, Schmainda KM, Bennett RT, Rowe DB, Lu H, Hyde JS. Characterization of continuously distributed cortical water diffusion rates with a stretched-exponential model. Magn Reson Med. 2003:50:727-34

14. Jensen JH, Helpern JA, Ramani A, Lu H, Kaczynski K. Diffusional kurtosis imaging: the quantification of non-gaussian water diffusion by means of magnetic resonance imaging. Magn Reson Med. 2005;53:1432-40.

15. Suo S, Cheng F, Cao M, Kang J, Wang M, Hua J. Multiparametric diffusion-weighted imaging in breast lesions: association with pathologic diagnosis and prognostic factors. J Magn Reson Imaging. 2017:46:740-50

16. Liu C, Wang K, Li X, Zhang J, Ding J, Spuhler K. Breast lesion characterization using whole-lesion histogram analysis with stretched-exponential diffusion model. J Magn Reson Imaging. 2018;47:1701-10.

17. Bickelhaupt S, Jaeger PF, Laun FB, Lederer W, Daniel H, Kuder TA. Radiomics based on adapted diffusion kurtosis imaging helps to clarify most mammographic findings suspicious for cancer. Radiology. 2018;287:761-70

18. Bahl M, Barzilay R, Yedidia AB, Locascio NJ, Yu L, Lehman CD. Highrisk breast lesions: a machine learning model to predict pathologic upgrade and reduce unnecessary surgical excision. Radiology. 2018;286:810-8.

19. Kniep HC, Madesta F, Schneider T, Hanning U, Schonfeld MH, Schon G Radiomics of brain MRI: utility in prediction of metastatic tumor type. Radiology. 2019;290:479-87.

20. Liu J, Sun D, Chen L, Fang Z, Song W, Guo D, et al. Radiomics analysis of dynamic contrast-enhanced magnetic resonance imaging for the prediction of sentinel lymph node metastasis in breast cancer. Front Oncol. 2019:9:980.

21. Liang C, Cheng Z, Huang Y, He L, Chen X, Ma Z. An MRI-based radiomics classifier for preoperative prediction of Ki-67 status in breast cancer. Acad Radiol. 2018;25:1111-7.

22. Chai $R, M a H, X u M$, Arefan D, Cui X, Liu Y. Differentiating axillary lymph node metastasis in invasive breast cancer patients: a comparison of radiomic signatures from multiparametric breast MR sequences. J Magn Reson Imaging. 2019;50:1125-32.

23. Truhn D, Schrading S, Haarburger C, Schneider H, Merhof D, Kuhl C. Radiomic versus convolutional neural networks analysis for classification of contrast-enhancing lesions at multiparametric breast MRI. Radiology. 2019;290:290-7.

24. Bonekamp D, Kohl S, Wiesenfarth M, Schelb P, Radtke JP, Gotz M. Radiomic machine learning for characterization of prostate lesions with MRI: comparison to ADC values. Radiology. 2018;289:128-37.

25. Dong Y, Feng Q, Yang W, Lu Z, Deng C, Zhang L. Preoperative prediction of sentinel lymph node metastasis in breast cancer based on radiomics of T2-weighted fat-suppression and diffusion-weighted MRI. Eur Radiol. 2018:28:582-91.

26. van Griethuysen JJM, Fedorov A, Parmar C, Hosny A, Aucoin N, Narayan V. Computational radiomics system to decode the radiographic phenotype. Cancer Res. 2017;77:e104-7.

27. Gu D, Hu Y, Ding H, Wei J, Chen K, Liu H. CT radiomics may predict the grade of pancreatic neuroendocrine tumors: a multicenter study. Eur Radiol. 2019;29:6880-90.

28. Breiman LML. Random forests. 2001;45:5-32.

29 Chen S-Y, Feng Z, Yi X. A general introduction to adjustment for multiple comparisons. J Thorac Dis. 2017:9:1725.

30. Abraham A, Pedregosa F, Eickenberg M, Gervais P, Mueller A, Kossaifi J. Machine learning for neuroimaging with scikit-learn. Front Neuroinform. 2014;8:14.

31. Woodard GA, Ray KM, Joe BN, Price ER. Qualitative radiogenomics: association between Oncotype DX test recurrence score and BI-RADS mammographic and breast MR imaging features. Radiology. 2018;286:60-70.

32. Bickelhaupt S, Paech D, Kickingereder P, Steudle F, Lederer W, Daniel H. Prediction of malignancy by a radiomic signature from contrast agentfree diffusion MRI in suspicious breast lesions found on screening mammography. J Magn Reson Imaging. 2017;46:604-16.

33. Bogner W, Pinker-Domenig K, Bickel H, Chmelik M, Weber M, Helbich TH. Readout-segmented echo-planar imaging improves the diagnostic performance of diffusion-weighted MR breast examinations at 3.0 T. Radiology. 2012;263:64-76.

34. Gruber S, Minarikova L, Pinker K, Zaric O, Chmelik M, Strasser B. Diffusion-weighted imaging of breast tumours at 3 Tesla and 7 Tesla: a comparison. Eur Radiol. 2016;26:1466-73.

35 Ehteshami Bejnordi B, Veta M, Johannes van Diest P, van Ginneken B, Karssemeijer N, Litjens $G$. Diagnostic assessment of deep learning algorithms for detection of lymph node metastases in women with breast cancer. JAMA. 2017:318:2199-210.

36. Xie T, Wang Z, Zhao Q, Bai Q, Zhou X, Gu Y, et al. Machine learningbased analysis of MR multiparametric radiomics for the subtype classification of breast cancer. Front Oncol. 2019;9:505.

37. Fan $M$, Zhang P, Wang Y, Peng W, Wang S, Gao X. Radiomic analysis of imaging heterogeneity in tumours and the surrounding parenchyma based on unsupervised decomposition of DCE-MRI for predicting molecular subtypes of breast cancer. Eur Radiol. 2019;29:4456-67.

38. Sutton EJ, Oh JH, Dashevsky BZ, Veeraraghavan $H$, Apte AP, Thakur SB. Breast cancer subtype intertumor heterogeneity: MRI-based features predict results of a genomic assay. J Magn Reson Imaging. 2015:42:1398-406

39. Kolarevic D, Tomasevic Z, Dzodic R, Kanjer K, Vukosavljevic DN, Radulovic M. Early prognosis of metastasis risk in inflammatory breast 
cancer by texture analysis of tumour microscopic images. Biomed Microdevices. 2015;17:92.

40. Waugh SA, Purdie CA, Jordan LB, Vinnicombe S, Lerski RA, Martin P. Magnetic resonance imaging texture analysis classification of primary breast cancer. Eur Radiol. 2016;26:322-30.

\section{Publisher's Note}

Springer Nature remains neutral with regard to jurisdictional claims in published maps and institutional affiliations.
Ready to submit your research? Choose BMC and benefit from:

- fast, convenient online submission

- thorough peer review by experienced researchers in your field

- rapid publication on acceptance

- support for research data, including large and complex data types

- gold Open Access which fosters wider collaboration and increased citations

- maximum visibility for your research: over $100 \mathrm{M}$ website views per year

At BMC, research is always in progress.

Learn more biomedcentral.com/submissions 